\title{
Jupiter's polar auroral emissions
}

\author{
D. Grodent, ${ }^{1}$ J. T. Clarke, ${ }^{2}$ J. H. Waite Jr., ${ }^{3}$ S. W. H. Cowley, ${ }^{4}$ J.-C. Gérard, ${ }^{1}$ and J. Kim ${ }^{2}$ \\ Received 2 May 2003; revised 17 June 2003; accepted 1 July 2003; published 18 October 2003.
}

[1] This paper reports a study of Jupiter's polar auroral emissions observed in an extended series of FUV images. They were obtained on seven days, during winter 2000-2001, with the STIS camera on board the Hubble Space Telescope. The fixed pointing yielded highly accurate and consistent tracking of emisson features as Jupiter rotated, allowing the analysis of the auroral morphology and brightness on timescales ranging from seconds to days. In the Northern Hemisphere, the polar emissions, located poleward of the main oval, usually represent about $30 \%$ of the total auroral FUV emitted power. They show emission bursts lasting $\sim 100 \mathrm{~s}$, while the main oval remains stable. The polar region may be divided into three regions apparently fixed in magnetic local time: the dawnside dark region, the poleward swirl region, and the duskside active region in which flares and arc-like features are observed. Each of these UV emission regions can be identified with its infrared counterpart and probably relates to a different sector of the Dungey cycle or Vasyliunas cycle plasma flows. INDEX TERMS: 6220 Planetology: Solar System Objects: Jupiter; 2704 Magnetospheric Physics: Auroral phenomena (2407); 2756 Magnetospheric Physics: Planetary magnetospheres (5443, 5737, 6030); 2784 Magnetospheric Physics: Solar wind/magnetosphere interactions; KEYWORDS: Jupiter, aurora, reconnection, planetary magnetospheres, solar wind/magnetosphere interactions

Citation: Grodent, D., J. T. Clarke, J. H. Waite Jr., S. W. H. Cowley, J.-C. Gérard, and J. Kim, Jupiter's polar auroral emissions, J. Geophys. Res., 108(A10), 1366, doi:10.1029/2003JA010017, 2003.

\section{Introduction}

[2] Hubble Space Telescope (HST) images have shown that Jupiter's aurora exhibits three distinct regions, based on their locations, the physical regions and processes from which they originate, and their independent variations with time [Grodent et al., 2003; Clarke et al., 1998]. These three regions (Figure 2) can be summarized as: (1) the satellite footprint emissions, (2) the main oval emissions, and (3) all other emissions poleward of the main oval, which we refer to as the "polar emissions." The satellite footprint aurora are easily identified by the fact that they remain fixed along magnetic flux tubes connected to Io, Europa, and Ganymede [Clarke et al., 2002]. The main oval emissions are observed to corotate with Jupiter [Ballester et al., 1996] and are relatively stable, exhibiting variations on time scales of tens of minutes to hours [Grodent et al., 2003]. By contrast, the polar emissions vary rapidly, up to the extreme cases represented by the "flares" [Waite et al., 2001], which can rise from the background level of a few kiloRayleighs $(\mathrm{kR})$ to several MR in brightness in tens of seconds. Three independent papers [Bunce and Cowley, 2001; Hill, 2001;

\footnotetext{
${ }^{1}$ LPAP, Institut d'Astrophysique et de Géophysique, Université de Liège, Belgium. USA.

${ }^{2}$ Center for Space Physics, Boston University, Boston, Massachusetts,

${ }^{3}$ Space Physics Research Laboratory, University of Michigan, Ann Arbor, Michigan, USA.

${ }^{4}$ Department of Physics and Astronomy, University of Leicester, Leicester, UK.
}

Copyright 2003 by the American Geophysical Union. 0148-0227/03/2003JA010017
Southwood and Kivelson, 2001] suggest that the main jovian auroral oval is connected with the magnetosphereionosphere coupling current system associated with the breakdown of rigid corotation in the middle magnetosphere region. The main auroral oval may thus result from the upward Birkeland current that enforces partial corotation of plasma moving outward from the Io plasma torus.

[3] In the Earth's aurora the area poleward of the main oval generally corresponds to open field lines. By contrast, a significant fraction of the poleward regions on Jupiter may correspond to closed field lines mapping to the outer magnetosphere, with a limited area of open field lines whose location is presently under debate. Therefore we refer to Jupiter's emissions poleward of the main oval generically as "polar emissions" so as to not confuse them with the polar cap at Earth.

[4] To some extent, the observed locations of auroral emissions can be combined with a magnetic field model to "map" the emissions to specific regions of the magnetosphere to determine the physical processes responsible for the particle acceleration and aurora. Such a mapping assumes knowledge from a reliable model of the path of magnetic field lines. In this work we applied the VIP4 magnetic model developed by Connerney et al. [1998]. The mapping rests on the principle that the farther the magnetospheric plasma from the planet, the closer to the magnetic pole the associated auroral emission. The accuracy of the mapping decreases as one approaches the magnetic pole where the field strength increases and field lines are closer together. Therefore a small latitudinal variation of the ionospheric end of a field line corresponds to an increasingly larger radial shift of the magnetospheric end of the 


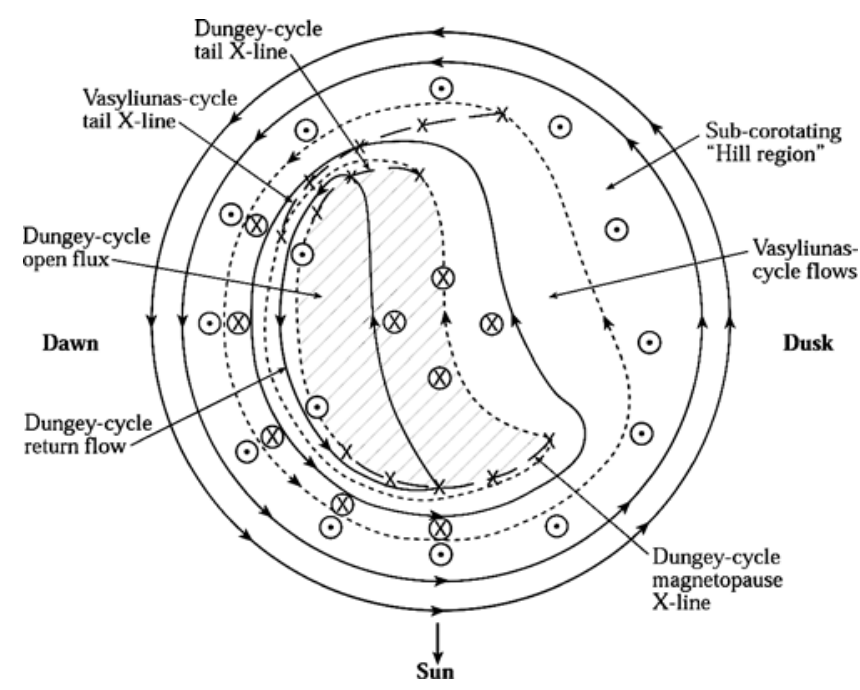

Figure 1. Sketch of the plasma flows in the northern jovian hemisphere and their relation to the plasma flows in the equatorial plane. The flows are shown in a frame of reference where the planetary dipole axis is at rest. Taken from Cowley et al. [2003].

field line. This uncertainty is magnified by the large azimuthal currents in the current sheet in Jupiter's middle magnetosphere, giving the local field a strong radial component which further stretches the radial distance between closely space field lines at the planet.

[5] Stallard et al. [2001] used spatially resolved Doppler shifts of spectral lines of the $\mathrm{H}_{3}^{+}$ion to derive a detailed infrared (IR) picture of the auroral morphology. Poleward of an auroral oval, matching the main oval observed in the UV images, they identified Dark and Bright Polar Regions (DPR, and BPR), corresponding to Satoh et al. [1996] yin-yang structure. Most recently, Stallard et al. [2003] found that the DPR may be divided into two separate regions: the fixed DPR (f-DPR), which is near stagnant in the magnetic pole reference frame (the MPRF of Stallard et $a l$. [2003], that is a frame oriented with respect to the sun but fixed to the rotating magnetic pole), and the rotating DPR (r-DPR), located between the f-DPR and the main oval in the dawn and noon sectors and which was found to subcorotate with the planet. Based on those IR observations, Cowley et al. [2003] proposed a general view of the equatorial and ionospheric plasma flows associated with the auroral emissions. Figure 1 (taken from Cowley et al. [2003]) shows a sketch of such plasma flows in the northern Jovian ionosphere, transformed to a frame where the planetary dipole axis is at rest, and their relation to the plasma flows in the equatorial plane of Jupiter. Three flow components are considered in this basic picture which, from lower to higher magnetic latitudes, are as follows. (1) The subcorotating "Hill region" associated with the breakdown of rigid corotation in the middle magnetosphere region and presumably giving rise to the main auroral oval as a result of the upward Birkeland current that enforces partial corotation of the outward moving iogenic plasma (see also Hill [1979]). (2) The Vasyliunas cycle flows which correspond to a subcorotating region where iogenic plasma is lost down tail, principally in the dusk and midnight sectors via reconnection across the current sheet, leading to the forma- tion of a tail X-line from which plasmoids are detached from the rotating plasma [Vasyliunas, 1983]. This region is narrowest in the dawn sector where empty flux tubes (which initially carried the detached plasmoid) return to near corotation. The field-aligned current in this sector is then directed downwards (upward moving electrons), such that it will be aurorally dark, while discrete emissions may occur in the dusk sector of this region associated with the plasmoid formation process. (3) The Dungey cycle flow region, located principally on the dawnside of the magnetosphere, which is associated with the solar wind interaction [Dungey, 1961]. It consists of a region of open field lines and anti-sunward flow, shown hatched in Figure 1, and a region of closed flux and return sunward flow taking place only on the dawnside of the region of open flux. The dayside reconnection associated with the solar wind-driven process lies roughly symmetrically with respect to noon, along the Dungey cycle magnetopause X-line represented in Figure 1. Since the region of open magnetic flux is magnetically connected to a tail lobe with very low plasma density and essentially no hot plasma, it is expected to be auroraly dark and almost stagnant in the corotating frame.

[6] On the basis of this picture, Cowley et al. [2003] suggested that the IR f-DPR may be identified with the stagnant open field region, while the r-DPR was interpreted as the sunward-flowing layer of return Dungey and Vasyliunas cycle flows in the dawn sector. Similarly, the BPR may correspond to the down-tail flow in the Vasyliunas cycle on the duskside and may also include the dayside auroral activity associated with the dayside magnetopause reconnection process. Though the correspondance between the UV and IR morphologies has not yet been demonstrated in detail by simultaneous observations, we will use the same general picture (Figure 1) in order to interpret the UV polar emissions.

\section{Observations}

[7] During the the winter of 2000-2001, the STIS camera on the HST obtained approximately 200 far-ultraviolet (FUV) images of the auroral emission at Jupiter's poles. These observations were completed directly before and after Cassini's closest approach of Jupiter on 30 December 2000. They span a period of 6 weeks starting on 14 December 2000 and ending on 21 January 2001 (see Grodent et al. [2003] for a full description of the data set). Apart from one occasion, the guide stars remained the same during each data set (each day). Therefore it is assumed that the pointing did not change between two groups of images taken on the same day (at the same pole). This entire data set represents a major improvement over previous observations, since the locations of auroral emission regions could be observed "continuously" for several hours.

[8] The images were taken with the photon-counting detector 25MAMA (Multi-Anode Micro channel Array) using the Clear aperture (no filter). In this mode, the solarblind detector has a bandpass ranging from 115 to $170 \mathrm{~nm}$ and is sensitive to the $\mathrm{H}_{2}$ Lyman and Werner bands as well as the strong H Lyman- $\alpha$ line. Most of the images were taken during dark time, that is the 45 -min period during which HST is in the shadow of the Earth, and therefore minimal contamination is expected from the geocoronal Lyman- $\alpha$ 


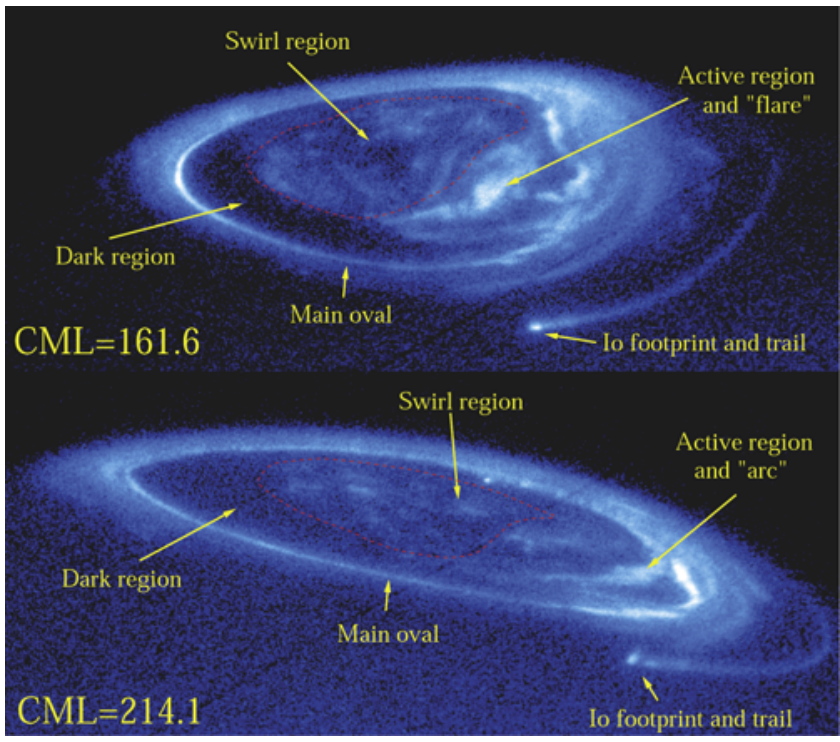

Figure 2. Raw HST-STIS images taken on 14 December 2000. The CML of the upper (Clear) image is $161.6^{\circ}$ and is $214.1^{\circ}$ for the lower (SrF2 filtered) image. These images illustrate the recurrent auroral features appearing in the north: the main oval, the Io footprint and its trail, and the rest of the emission poleward of the main oval, that we refer to as the polar aurora. The differences between the two images are typical of the variability of the polar emissions in a 1.5 hours time period. Arrows point to the dark region and the active region where "flares" and "arcs" are often observed. The dashed contour limits the swirl region discussed in section 4.2. The polar projections of these two images appear in Figure 6.

emission. In addition to the accumulated Clear images, a series of time tagged images were taken with the SrF2 filter which cuts out most of the emission shortward of $\sim 130 \mathrm{~nm}$, including the Lyman- $\alpha$ emission. The Clear and filtered images show essentially the same auroral features (Figure 2). The MAMA array consists of $1024 \times 1024$ pixels providing a field of view (FOV) of $24.7^{\prime \prime} \times 24.7^{\prime \prime}$ with a $\sim 0.08^{\prime \prime}$ full width at half maximum point spread function (PSF). For a direct comparison, the images were all scaled in pixel size to display Jupiter as it would appear at a distance of 4.2 Astronomical Units (AU). The distance subtended by one pixel on the field of view projected at Jupiter is then $\sim 74 \mathrm{~km}$. At that distance, a resolution element corresponds to $\sim 300 \mathrm{~km}$ on the planet. All the (Clear) images were accumulated for $\sim 100 \mathrm{~s}$, during which Jupiter rotates by $\sim 1^{\circ}$. This rotation introduced a faint smearing of the images which, for a surface feature located at the central meridian longitude (CML) and at $60^{\circ}$ latitude (a severe case), is of the order of 8 pixels, that is approximately two times the PSF. The blurring is relatively less as one approaches the east or west limb, where the rotational motion is more along the line of sight. All auroral images were reduced from the initial data files using the procedure described by Clarke et al. [2002] and Grodent et al. [2003]. The brightnesses derived in this paper assume a conversion factor of 0.0013 count per pixel per second for 1 kiloRayleigh $(\mathrm{kR})$ of $\mathrm{H}_{2}$ emission plus Lyman- $\alpha$ for the images using the FUV-MAMA clear bandpass [Clarke et al., 1998], and 0.0005 count per pixel per second per kR for the FUV-MAMA images using the $\mathrm{SrF} 2$ filter.

[9] The viewing geometry for the southern aurora is less favorable for Earth-based observations of Jupiter's aurora. The proximity of the magnetic south pole to the rotation axis restricts the view of the auroral distribution compared with the northern aurora. Moreover, due to the subsolar longitude and latitude, most of the southern limb was not illuminated by the Sun and did not provide a sharp limb. Therefore the limb-fitting accuracy in the south is more uncertain. In any case, the stretching of the southern emission near the limb persists and the location of the auroral emission in the south remains less accurate. For this reason, we put the emphasis of this paper on the images taken in the north. Animations based on image sequences and time tagged images obtained during the Cassini-Jupiter flyby are available online at the LPAP web site (Université de Liège) (available at http:// lpap.astro.ulg.ac.be/jupiter).

\section{Auroral Emitted Power}

[10] The main oval usually contributes $\sim 70 \%$ of the total auroral emission [Grodent et al., 2003]; the rest is mainly due to the polar region (i.e., the region poleward of the main oval). The brightenings that are often observed in the polar region (the flares) slightly modify this ratio, though exceptional events such as the one described by Waite et al. [2001] contribute more significantly to the total emission. Figure 3 presents the total emitted power, above the jovian disk background in the auroral region, as a function of time in the Northern Hemisphere. Three time tagged images were considered with a time resolution of $5 \mathrm{~s}$. The first one (Figure 3a) was taken on 16 December 2000 when the CML was $127^{\circ}$ (all longitudes are given in System III coordinates [S3]), the second one was obtained on 14 December 2000 (Figure 3b) with $\mathrm{CML}=178^{\circ}$, and the last one (Figure 3c) was acquired on 18 December 2000 at $\mathrm{CML}=241^{\circ}$. It is immediately clear that the short time variations $(100 \mathrm{~s})$ of the emitted power integrated over the whole emission region (excluding the Io footprint and its trail) are mainly due to the polar emissions. Indeed, the curves showing the power emitted in the main oval are almost flat. The slopes of the three curves, $\sim 5 \%$ increase in Figure $3 a$, slightly positive in Figure $3 b$, and $\sim 5 \%$ decrease in Figure $3 \mathrm{c}$, are consistent with the variation of the area of the visible auroral region as the planet rotates. If one considers the area poleward of the main oval with the December 2000 observing geometry then Figure 4 shows that, over $300 \mathrm{~s}$, the visible area increases by $\sim 4 \%$ at $\mathrm{CML}$ $\sim 130^{\circ}$, increases by $\sim 0.3 \%$ at $\mathrm{CML} \sim 180^{\circ}$, and decreases by $\sim 4 \%$ at $\mathrm{CML} \sim 240^{\circ}$. These trends are noticeable in the curves showing the power emitted in the polar region. However, for these curves the major fluctuations are on the order of $10 \%$ and take the form of well defined bursts lasting about $100 \mathrm{~s}$. Figure 4 also suggests that while the areas at $\mathrm{CML} \sim 130^{\circ}$ and $\sim 240^{\circ}$ are about $60 \%$ of the area at $\sim 180^{\circ}$, the corresponding power ratios deduced from Figure 3 are on the order of $30 \%$. Therefore geometrical factors contribute only a fraction of the variation of the power emitted in the polar region. A thorough analysis of the main oval power curves indicates faint bumps associ- 

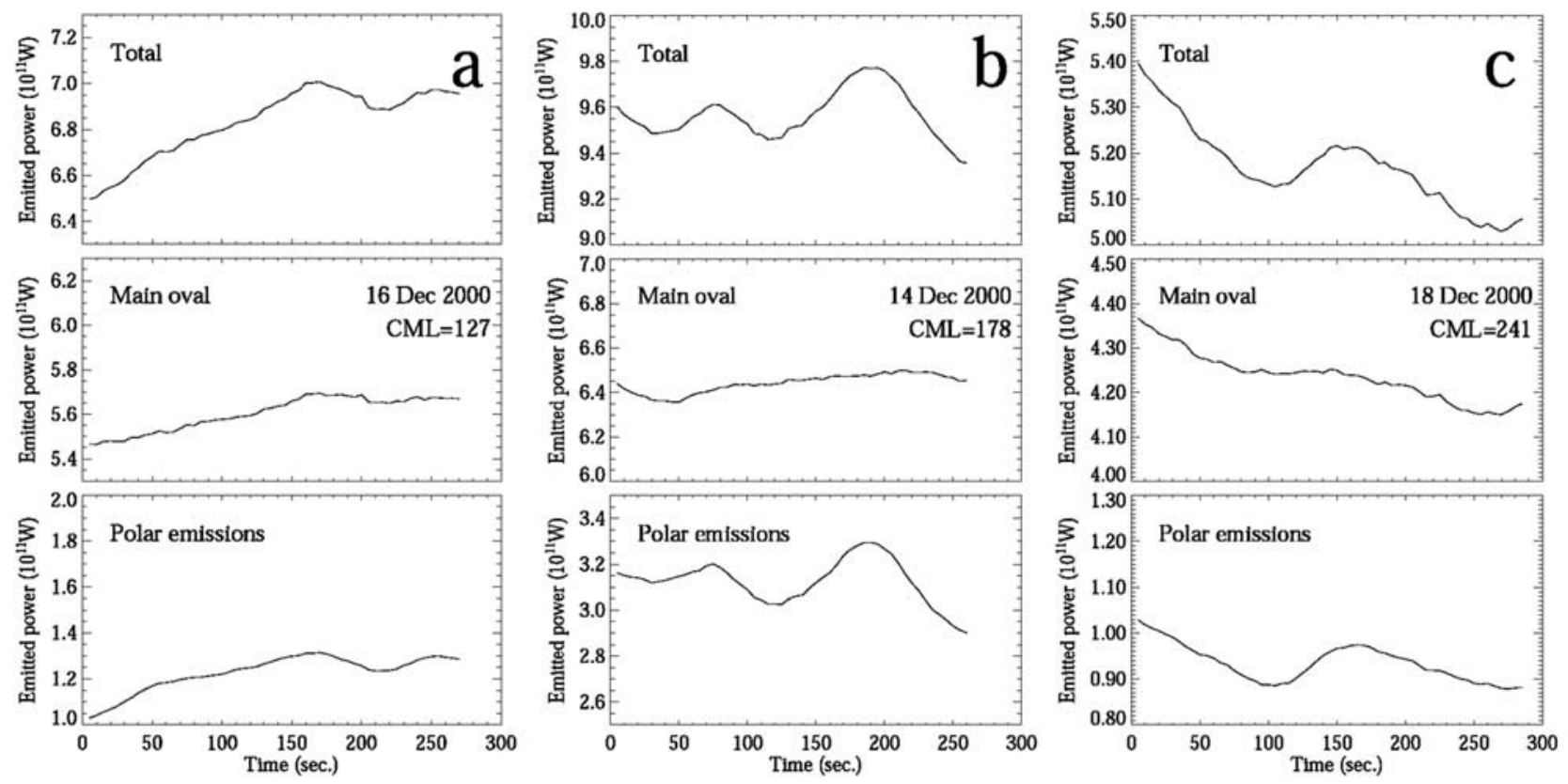

Figure 3. Total emitted power as a function of time in the north polar region (lower panel), in the main oval (middle panel), in both (top panel). Three time tagged images were considered with a time resolution of $5 \mathrm{~s}$ and a smoothed over $45 \mathrm{~s}$ with a boxcar average. Figure $3 \mathrm{a}$ corresponds to an image taken on 16 December 2000 (1125:51 UT) when the CML was $127^{\circ}$ (S3). Figure 3b was obtained on 14 December $2000\left(1112: 02\right.$ UT) with $\mathrm{CML}=178^{\circ}$, and Figure 3c was acquired on 18 December 2000 (1610:29 UT) at $\mathrm{CML}=241^{\circ}$. Note that in Figure $3 \mathrm{c}$ the power scales have been halved compared with Figures 3a and $3 \mathrm{~b}$. It is clear that the short time variations $(100 \mathrm{~s})$ of the emitted power integrated over the whole emission region (excluding the Io footprint and its trail) are mainly due to the polar emissions.

ated with the polar bursts. They likely stem from the vertical extent of the polar emission near the limb which, as a result of limb brightening [Grodent et al., 1997], contaminates the main oval emission at the limb. Figure 3 thus clearly demonstrates that the polar emissions are far more variable than the main oval emission, the former being characterized by brightenings lasting $\sim 100 \mathrm{~s}$. For the three cases described here, their contribution to the total emitted power varies from 20 to $35 \%$. These observations suggest that the main oval emission is decoupled from the polar emissions which indicates that they probably stem from different flow dynamics, although the same precipitation mechanism [Knight, 1973] may apply to both emissions. This decoupling is further suggested by FUV spectral observations [Gérard et al., 2003] which show that the rapid brightenings observed in the high-latitude emission (that we refer to as bursts) are not correlated with enhancements of the main oval in the same longitude sector.

\section{Polar Auroral Regions}

[11] Few reports have been published about the detailed characteristics of the polar FUV emissions. Compared with the main auroral oval, these emissions map along magnetic field lines further out in the magnetosphere at radial distances greater than $30 \mathrm{R}_{J}$. Owing to field distortions induced by the current sheet and subsequent magnetic field model uncertainty, it is more difficult to determine the specific magnetospheric source region associated with the polar emissions. Note that in comparison with the Earth, where regions poleward of the main auroral oval are generally on open field lines, at Jupiter the main oval maps between inner distances of $\sim 20 R_{J}$ and outer distances of several tens of $\mathrm{R}_{J}$.

[12] Figure 5 highlights three regions showing different sorts of emission identified by their average brightness and dynamical behavior: the dark region (yellow contour), the swirl region (red contour), and the active region (green contour). The shape and position of the three regions are shown at four different CMLs. They were

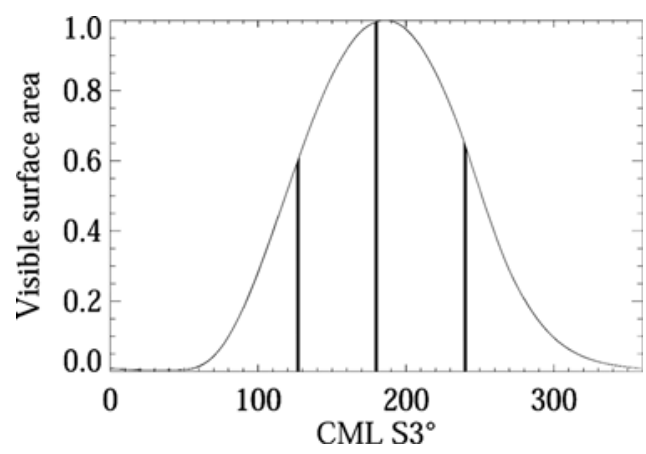

Figure 4. Surface area (normalized to the maximum value) limited by the main oval as a function of the CML, assuming the viewing geometry of December 2000. The grey vertical bars mark the $3^{\circ}$-wide CML ranges around $127^{\circ}, 178^{\circ}$, and $241^{\circ}$, corresponding to the midexposure CMLs considered in Figure 3. 

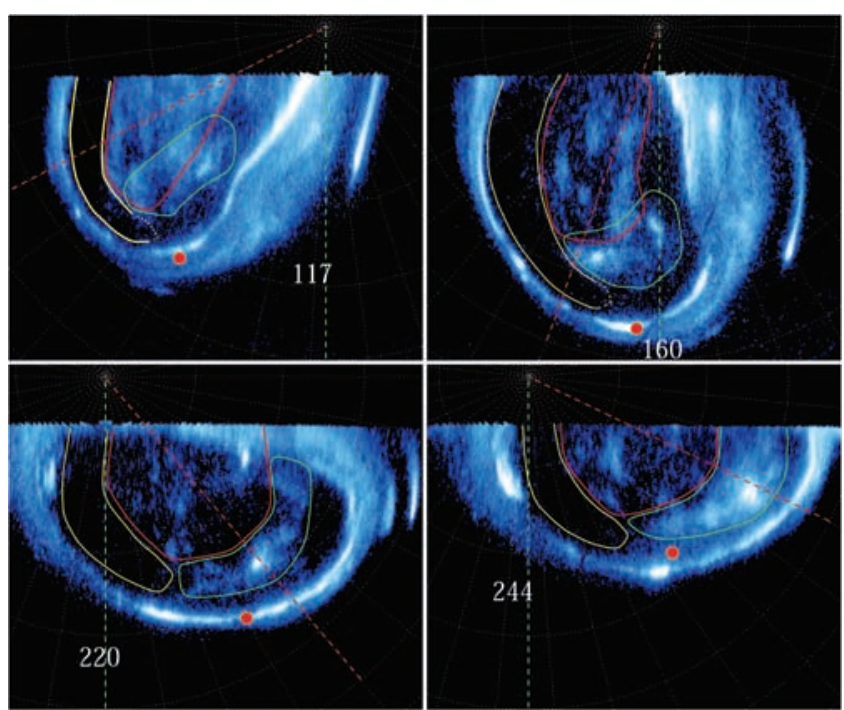

Figure 5. Polar projections of the northern auroral region showing the shape and position of the dark region (yellow contour), the swirl region (red contour), and the active region (green contour) as they appear at $\mathrm{CML}=117,160$, 220 , and 244 degrees. The eastern end of the dark region appears dotted where it is not sharply defined. Parallels and meridians are drawn every $10^{\circ}$. The CML is marked with a vertical green dashed line and longitude $180^{\circ}$ is highlighted with a red dashed line. The red dots locate the magnetic footprint of Ganymede (VIP4 model) as the orbital longitude of the satellite matches the CML and therefore indicates the direction of magnetic noon at $15 \mathrm{R}_{J}$. The four images were taken from the 16 December 2000 subset. The instantaneous limits of the regions were determined by hand from the brightness distribution and dynamical behavior observed in image sequences including those presented in the figure: six images plus one time-tagged image around $\mathrm{CML}=117^{\circ}$, three images around $160^{\circ}$, eight images around $220^{\circ}$, and two images plus one time-tagged image around $\mathrm{CML}=244^{\circ}$.

determined from image sequences and time tagged images which allowed us to follow the morphological and brightness variations of the polar emissions as a function of time. These three regions also appear clearly in the two unprojected images shown in Figure 2.

[13] In Figure 5 the theoretical location of Ganymede's magnetic footprint is used to obtain a proxy for the direction of the magnetic noon meridian (at $15 \mathrm{R}_{J}$ ). The footprint location corresponding to magnetic noon is obtained from the VIP4 model in which we assumed that the satellite lies in the plane of the central meridian, i.e., when the orbital longitude of the satellite intersects the CML. With this landmark overlaid on the figure as the red dot, the three polar regions clearly appear fixed in magnetic local time (MLT), that is these auroral regions may be magnetically connected to magnetospheric processes occuring at fixed local times.

\subsection{Dark Region}

[14] The dark region (in the north) may be described as a dawnside crescent-shaped region almost devoid of auroral emission (yellow contour in Figure 5). It is limited by the main oval on the equatorward edge and by the swirl and active regions, described below, on the poleward edge. As illustrated by Figure 5, the boundary of the dark region in the different CML ranges is subcorotating, i.e., an observer above the north pole, fixed relative to the direction of the Sun, would see the dark region confined to the dawn to noon sector, as the CML increases from $110^{\circ}$ to $270^{\circ}$. The general shape of the dark region does not change much either, it just appears to be stretched or compressed according to the direction of the major axis defining the main oval which is corotating with the planet. At CMLs smaller than $\sim 180^{\circ}$ (upper panels of Figure 5), the eastern end of the dark region (dotted portion of the yellow contour) is not sharply defined and depends on the emission gradient in the region between the equatorward edge of the active region and the dusk portion of the main oval. This latter region may be seen as the duskside portion of the Vasyliunas cycle flows where previously emptied flux tubes are gradually mass loaded with iogenic plasma. It is characterized by faint emission on the order of a few tens of $\mathrm{kR}$ above the disk background, corresponding to an electron energy precipitation of a few $\mathrm{mW} \mathrm{m}^{-2}$. At CMLs greater than $\sim 180^{\circ}$ (lower panels of Figure 5), the active region moves equatorward and provides a sharper boundary for the eastern end of the dark region. Nevertheless, it appears that this end is almost fixed relative to the magnetic noon meridian. Such behavior is characteristic of a region fixed in MLT.

[15] Based on morphological similarities, the UV dark region may be identified with the rotating Dark Polar Region (r-DPR) deduced from ground-based Doppler observations in the infrared [Stallard et al., 2001, 2003]. Like the UV dark region, this IR region is adjacent to the poleward edge of the main oval in the dawn sector. The ionospheric plasma within it is found to flow sunward at subcorotational speeds. Cowley et al. [2003] suggested that the subcorotating IR r-DPR, wich we now identify with the UV dark region, may plausibly be connected with the partially emptied flux tubes in the sunward return flows associated with the Dungey and Vasyliunas cycles (Figure 1).

[16] Since the field-aligned currents connecting to this region have been suggested to be downward directed (upward moving electrons) it is expected that this region is almost auroraly dark. Indeed, further analysis of the UV dataset shows that the dark region is filled with weak emission ranging from 0 to $10 \mathrm{kR}$ above the planetary disk background, the smaller values being found in images at CML smaller than $150^{\circ}$. Application of the energy degradation model described by Grodent et al. [2001] shows that this emission correponds to the precipitation of electrons (assuming that upward and downward moving electrons give rise to the same height-integrated effects) having an energy flux of 0 to $1 \mathrm{~mW} \mathrm{~m}^{-2}$, consistent with $\mathrm{H}_{3}^{+}$emission ranging from 0 to $0.1 \mathrm{~mW} \mathrm{~m}^{-2}$. This latter number is one order of magnitude smaller than the one given by Stallard et al. [2001], who showed that the IR DPR intensity is typically $30-40 \%$ of that of the auroral oval, that is about $1 \mathrm{~mW} \mathrm{~m}^{-2}$ of $\mathrm{H}_{3}^{+}$emission (the f-DPR and r-DPR cannot be distinguished by their level of emission).

[17] The marginal energy flux deduced from the faint UV emission in the f-DPR suggests that auroral precipitation cannot explain the excess of IR emission. Stallard et al. 
[2003] suggested that the limited lifetime of $\mathrm{H}_{3}^{+}(<1000 \mathrm{~s})$ would prevent transport of $\mathrm{H}_{3}^{+}$from the bright regions to the DPR. Therefore other processes need to be investigated to explain the IR emission in the DPR.

\subsection{Swirl Region}

[18] In the north the swirl region (red contour in Figure 5) may be described as a region of faint, patchy, and shortlived (tens of seconds) emission features characterized by turbulent motions that occasionally form localized clockwise swirls (in the unprojected time tagged images), though the sense of rotation is not clear and varies from one dataset to the other. It appears clearly in the two unprojected images presented in Figure 2. It is located around the center of the polar region and fills approximately one third of the area limited by the main oval. Its dawn to noon flank is clearly limited by the poleward edge of the dark region while the noon to dusk flank is partially limited by the active region. In the latter sector, the upper panels of Figure 5 show that the swirl and active regions overlap when the CML is smaller than $\sim 180^{\circ}$. It should be noted again that the discrimination between the different regions rests not only on the different morphologies but also on the dynamical behaviors observed in image sequences and time tagged images. At higher CMLs (lower panels of Figure 5) the active region is shifted equatorward and the two regions are better discriminated, though some overlapping remains. The swirl region is a relatively small region approximately centered on the magnetic pole. It is difficult to follow the motion of the faint patchy short lived features that fill the swirl region. Therefore one cannot determine to what extent it is corotating (or not) with the bulk of the auroral emission. Its shape slightly varies with CML as a function of the stretching of the dark region and of the equatorward motion of the active region.

[19] The location and shape of the UV swirl region matches well the IR fixed Dark Polar Region (f-DPR) observed by Stallard et al. [2003] which is described as a region in which the plasma is near-stagnant with respect to the magnetic pole (that is in a frame where the dipole axis is at rest). Cowley et al. [2003] suggested that the location and the stagnant nature of the f-DPR may be associated with the region of open magnetic flux mapping to the tail lobes which is associated with the solar wind-driven Dungey cycle (hatched region in Figure 1). The low flow speeds are then consistent with the expected long ( $\sim 5-10$ day) residence times of open flux tubes in the lobes. The region of open flux should be aurorally "dark," since it is magnetically connected to a tail lobe with very low plasma density and essentially no hot plasma and with downward directed field-aligned currents (upward moving electrons). However, on average this region contributes half of the total polar UV emission. It is characterized by variable emission ranging from 0 to $200 \mathrm{kR}$ above the planetary disk background. Similar to the dark region, the energy degradation model translates this UV brightness to an electron energy flux of 0 to $20 \mathrm{~mW} \mathrm{~m}^{-2}$, giving rise to $\mathrm{H}_{3}^{+}$emission ranging from 0 to $2 \mathrm{~mW} \mathrm{~m}^{-2}$, in close agreement with the $\mathrm{H}_{3}^{+}$ emission determined by Stallard et al. [2001], which they found to range from 0.5 to $1.5 \mathrm{~mW} \mathrm{~m}^{-2}$ (it should be noted that the the $\mathrm{H}_{3}^{+}$emission has never been observed to drop below the $0.3-0.4 \mathrm{~mW} \mathrm{~m}^{-2}$ level in any part of the auroral region). Accordingly, the $\mathrm{UV}-\mathrm{H}_{2}$ and $\mathrm{IR}-\mathrm{H}_{3}^{+}$emissions appearing in the swirl region or f-DPR likely result from collisions with upward moving electrons, though it is not clear why these electrons should be accelerated up to the $\mathrm{H}_{2}$ ionization threshold energy $(15.4 \mathrm{eV})$. If this region is indeed the region of open flux, then the origins of the precipitation leading to the $\mathrm{H}_{2}-\mathrm{UV}$ and $\mathrm{H}_{3}^{+}$emissions remains to be determined.

\subsection{Active Region}

[20] The active region (green contour in Figure 5) includes two types of apparently related emission features: bright transient events, that we refer to as the polar flares and which give rise to the bursts observed in Figure 3, and a persistent arc-like feature. As will be discussed below and is readily apparent in Figure 5, the position and extent of the arc-like feature shape the contour of the active region and give it a subcorotational nature. Comparison with the magnetic noon mark (red dot in Figure 5) shows that this region is confined to the noon to postnoon sector (as previously suggested by Pallier and Prangé [2001]). Extending the identification of the UV and IR regions suggests that the IR Bright Polar Region (BPR) includes the active region plus the faint region extending between the equatorward edge of the swirl and active regions and the dusk portion of the main oval. In Figure 1 the latter region would then correspond to the down-tail flow in the Vasyliunas cycle while the active region would be connected to the Dungey cycle magnetopause X-line, that is the site of dayside reconnection of the jovian magnetic field lines with the interplanetary magnetic field (IMF) (it should be noted that the noon features discussed by Pallier and Prangé [2001] were also suggested to be related to the cusp).

\subsubsection{Polar Flares}

[21] An auroral flare developed inside the active region of the north pole on 14 December 2000 (upper panel of Figure 2) similar to, but much fainter than, the extremely bright emission observed with STIS on 21 September 1999 [Waite et al., 2001]. The flare appeared in a region located around S3 longitude $165^{\circ}$ and latitude $62^{\circ}$. Figure 6 shows nine consecutive images taken at $\mathrm{CML}$ ranging from $161^{\circ}$ to $214^{\circ}$. Note that the upper left and lower right projections correspond to the two images shown in Figure 2. The auroral flare is highlighted with a yellow oval (a) in the top left panel of Figure 6 . The emission extending from this feature is highlighted with a red comma-shaped contour (b). The vertical brightness of the flare (corrected for limb brightening with a cosine function) increased from $200 \mathrm{kR}$ to $400 \mathrm{kR}\left(C M L=167.6^{\circ}\right)$ then started to decrease and finally vanished, as illustrated by the quasi-empty yellow dotted oval $\left(\mathrm{a}^{\prime \prime}\right)$ at $C M L=209.4^{\circ}$ (it should be noted that the $\mathrm{a}^{\prime}$ feature was still present in an intermediate time tagged image taken at $\mathrm{CML}=178.3^{\circ}$ (not shown in Figure 6)). The brightness reached a peak value in $\sim 10 \mathrm{~min}$ and decreased to the initial value in a similar time. It then continuously decreased to the background level (few $\mathrm{kR}$ ) in $\sim 15 \mathrm{~min}$. These characteristic times are much longer (one order of magnitude) than those observed for the September 1999 flare [Waite et al., 2001]. The emission peaked when the flare was located near the MLT-noon meridian (derived from the VIP4 model at $15 \mathrm{R}_{J}$ ). Figure 6 also shows the local time motion of the auroral emission. It appears that the 
North polar region on 14 Dec. 2000
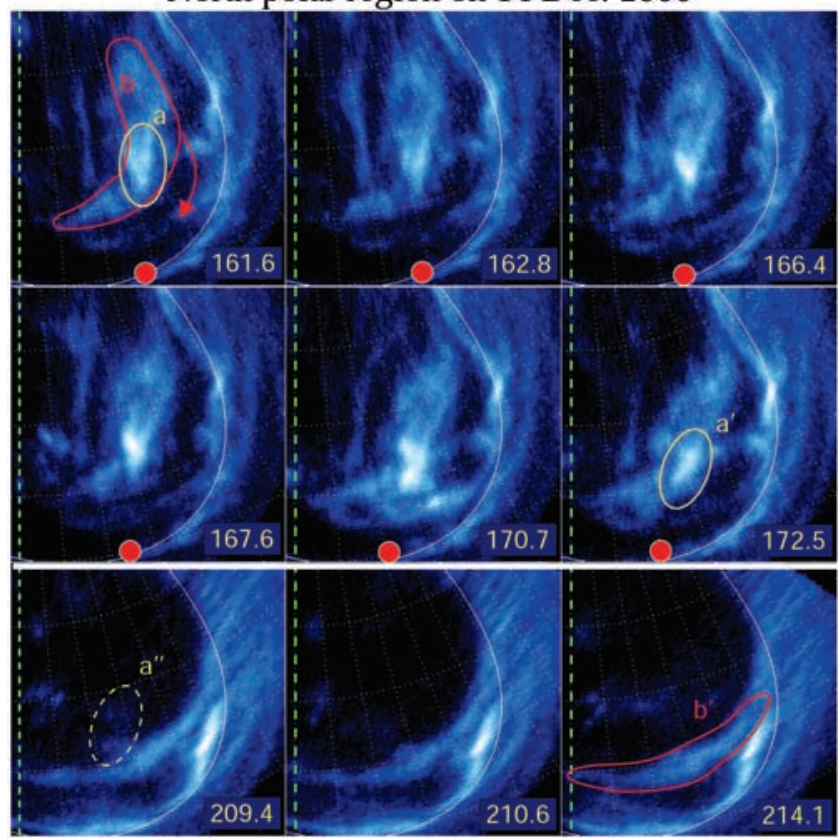

Figure 6. Polar projection of nine consecutive images taken on 14 December 2000 showing the evolution of a faint polar flare in the north ( $a \rightarrow a^{\prime} \rightarrow a^{\prime \prime}$, in yellow). The number in each panel's corner represents the CML in S3 degrees. The arc-like feature, marked with the red contour (b), is shifting equatorward $\left(\mathrm{b}^{\prime}\right)$ as the CML increases from 161.6 to $214.1^{\circ}$. A $5^{\circ}$ spaced grid and the main auroral oval are displayed on each panel. The vertical green dashed lines visualize the $180^{\circ}$ (S3) meridian and the lowest displayed parallel is $55^{\circ}$. The thick horizontal white bar marks the time gap $(\sim 1$ hour) between the six upper panels and the three lower panels. For the six upper panels, the red dot indicates MLT-noon as determined from the VIP4 magnetic model at the orbit of Ganymede $\left(15 \mathrm{R}_{J}\right)$. For the three lower panels the red dot is located at a S3 longitude of about $185^{\circ}$ and could not be displayed.

weight of the brightness distribution in the flare subcorotates and shifts equatorward by $\sim 5^{\circ}$ in 1.3 hours $(a \rightarrow$ $\left.a^{\prime} \rightarrow a^{\prime \prime}\right)$. It is remarkable to note that the September 1999 and December 2000 flare events occurred at similar locations. Moreover, they developed in the same magnetic local time region. Other flares have been frequently detected in the past [Clarke et al., 1998] such as, for instance, on 26 November 1998 at $168^{\circ}(S 3), 64^{\circ}$ north, again the same location. This flare developed when the $\mathrm{CML}$ was $164^{\circ}$, that is when the flare was mapping to the same region. The bright emissions observed with the FOC, at a lower spatial and temporal resolution, on 9 February 1992, 24 June 1994, and 5 September 1996 [Pallier and Prangé, 2001] may also be related to the polar flares. These bright features were observed between 1000 and 1200 MLT. These similarities suggest that the emission in the active region may be triggered by local time processes occurring in the vicinity of the noon sector of the magnetosphere, presumably near the magnetopause (that is, farther than $60 R_{J}$ ). However, it is important to stress that there may be a strong CML range selection effect to seeing the flares near noon, when they are probably more distributed. The strong variability of the flares, characterized by short time scales (minutes) does not support a slower solar wind controlled process, as would be expected if the flaring emissions were filling the polar cusp region. Instead, these rapid events may be a signature of an explosive reconnection with the IMF at the dayside magnetopause. While it seems increasingly likely that the bright auroral features (flares) discussed above are magnetically connected to the boundary of the dayside magnetosphere, very little has been said about Jovian dayside reconnection. Analysis of Voyager data during their entry into the Jovian magnetosphere [Huddleston et al., 1997] revealed bursty reconnection phases with the IMF characterized by flux transfer event (FTE) structures with timescales less than $1 \mathrm{~min}$. It was suggested that if the IMF B direction is at a favorable angle to the magnetospheric field, very sudden dynamic pressure jumps could lead to bursty reconnection events, allowing solar wind momentum to be transmitted into the magnetosphere. Walker and Russell [1985] found 14 possible FTEs in the Pioneer 10 and 11 and Voyager 1 and 2 observations of the Jovian magnetic field. Most of them were less than $1 \mathrm{~min}$ in duration and four of them were separated by about $4 \mathrm{~min}$. These timescales are in agreement with the characteristic times of the flaring emissions observed in the FUV images.

[22] A statistical analysis of the northern dataset shows that the active region is permanently filled with a variable amount of auroral emission. Figure 7 displays the power emitted in the active region as a function of CML from day to day during the campaign. With an average power of $1.5 \times$ $10^{11} \mathrm{~W}$, the data points taken on 14 December 2000 at a CML close to $170^{\circ}$, corresponding to the flare described above, dominate the present observation campaign by at least a factor of three. The two data points around CML $210^{\circ}$ are significantly lower and give an indication of the decreasing phase that followed the flare over a 1-hour interval. The 28 December 2000 and 13 January 2001 data points appear at an average emitted power of $10^{11} \mathrm{~W}$ while the rest of the data set remains around $0.4 \times 10^{11} \mathrm{~W}$ suggesting a moderate flaring activity on these 2 days and almost no activity during the rest of the time. The minimum and maximum powers measured in time-tagged data (data points joined with a vertical line in Figure 7) illustrate the level of fluctuation of the emission during the exposure time of a time tagged image. On 28 December 2000 the power fluctuated by a factor of two, that is a variation of $\pm 50 \%$ around the average value. The histogram plotted in the upper left corner of Figure 7 shows the percent of time the active region emissions, averaged over the exposure time, are in a certain power range (the duty cycle). It appears that for almost $55 \%$ of the observing time (approximately 1 hour) the emission is between 0 and $0.5 \times 10^{11} \mathrm{~W}$. It exceeds $1.5 \times 10^{11} \mathrm{~W}$ during about $7 \%$ of the time $(\sim 12$ minutes). This suggests that during the present campaign the probability for observing a relatively bright auroral flare $\left(\geq 1.5 \times 10^{11} \mathrm{~W}\right)$ in the north was less than $10 \%$, while there was a $30 \%$ chance to observe a moderate flare $(0.5-1.5 \times$ $\left.10^{11} \mathrm{~W}\right)$.

[23] The analysis of spectral observations obtained with STIS [Gérard et al., 2003] shows that the electron energies during the brightenings observed in the high latitude emission (the polar flares) typically range from 40 to $120 \mathrm{keV}$, 


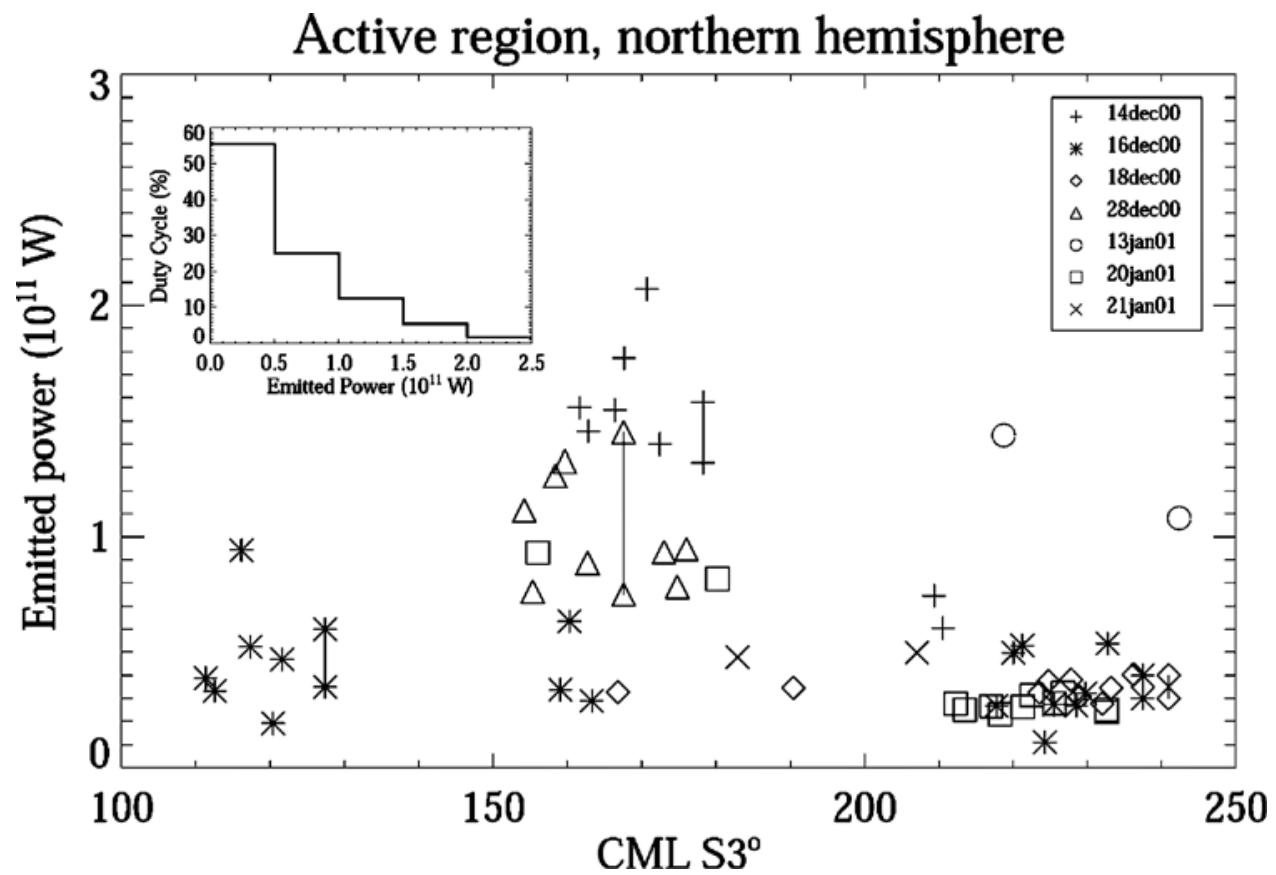

Figure 7. Power emitted in the active region (in the north) as a function of the CML. Different observation days are represented with different symbols. Emitted power ranges deduced from time tagged images are represented by two symbols joined by a vertical line. They give an indication of the time variation of the emission. The histogram in the upper left corner gives the duty cycle of the polar flares, i.e., the percent of time the active region emissions (averaged over the exposure time) are in a certain power range. A polar flare occurred on 14 December 2000 (plus symbol) when the CML was around $\lambda_{I I I} \sim 170^{\circ}$.

close to the values in the main oval. The time evolution of this energy shows little correlation or anticorrelation with the energy flux precipitated during these transient events. Gérard et al. [2003] therefore suggest that compared to the main auroral oval emission, the mechanism responsible for the flares does not increase the energy of the precipitated electrons but it enhances their number flux.

\subsubsection{Arc-Like Features}

[24] A polar feature resembling an auroral arc (b feature in Figure 6 and lower panel of Figure 2) is commonly observed around the flares discussed above. We shall refer to it as an "arc-like feature." Actually the distinction between the two types is rather morphological and it is likely that they stem from related, if not the same, magnetospheric pocesses. As already stated in section 4.3.1, Cowley et al. [2003] suggested the presence of a reconnection line which may take the form of an arc extending poleward of the main oval (the Dungey cycle magnetopause $\mathrm{X}$-line shown in Figure 1), near noon, and may therefore correspond to the arc-like feature described here. The equatorward motion of the arc-like feature, combined with an anticorotation slippage is illustrated in Figure $6\left(b \rightarrow b^{\prime}\right)$. It usually starts as an arc almost parallel to the (S3) longitude $170^{\circ}$ and ends as an arc aligned along the $65^{\circ}$ parallel. This equatorward motion was already observed in WFPC-2 images [Clarke et al., 1998] and was called the "equatorward surge." However, the limited sensitivity and angular resolution of WFPC-2 prevented one from pinpointing the subcorotation slippage. This slippage modifies the limit of the dark region and provides it a subcorotation "motion." This dayside equatorward motion might be seen as the ionospheric counterpart of the compression of the dayside magnetosphere that "squeezes" and accelerates the plasma. The arc-like feature is probably related to the "transpolar emission" [Pallier and Prangé, 2001] detected at a lower sensitivity along or just poleward of the main oval in the $150-160^{\circ} \mathrm{S} 3$ sector. Pallier and Prangé [2001] suggest that this feature may be related to the cusp, analog to what is observed in the Earth's dayside aurora, or an auroral flare similar to that described by Clarke et al. [1998].

\section{Summary}

[25] This paper reports the results of an imaging study of Jupiter's FUV polar aurora, observed with STIS between 14 December 2000 and 21 January 2001. For the first time, we provide a detailed description of the auroral emissions poleward of the main oval, referred to as the polar emissions. We relate them to the theoretical frame described by Cowley et al. [2003] which was originally based on the observation of $\mathrm{H}_{3}^{+}$auroral infrared emissions [Stallard et al., 2001, 2003] in Jupiter's northern hemisphere.

[26] 1. The polar emissions are shown to contribute $\sim 30 \%$ of the total auroral emission in the northern hemisphere and show emission bursts lasting $\sim 100 \mathrm{~s}$.

[27] 2. Based on morphological and dynamical considerations, the polar emissions are distributed over three regions apparently fixed in MLT: the dark region, the swirl region, and the active region. 
[28] 3. The dark region appears as a crescent-shaped region almost devoid of auroral emission $(<10 \mathrm{kR})$. It is adjacent to the poleward edge of the main oval in the dawn sector. This region may be identified with the infrared r-DPR zone and is probably connected with the return flows associated with the Dungey and Vasyliunas cycles, during which plasmoids are released down the tail. The dark region continues duskward with a region of faint emission (tens of $\mathrm{kR}$ ) which may be seen as the duskside portion of the Vasyliunas cycle flows where previously emptied flux tubes are gradually mass loaded with iogenic plasma.

[29] 4. The swirl region is a region of faint patchy emission features $(<200 \mathrm{kR})$ occasionally forming swirls. It is located around the center of the polar region and matches the near-stagnant f-DPR region observed in infrared emissions. It may correspond to the region of open magnetic flux which is associated with the solar winddriven Dungey cycle.

[30] 5. The active region is confined to the noon sector of the polar region, between the swirl region and the main oval. It includes two kinds of related features: the polar flares and the arc-like feature. This region may be seen as one component of the BPR observed in IR and is likely connected to the Dungey cycle magnetopause X-line, that is the site of dayside reconnection of the jovian magnetic field lines with the IMF.

[31] 6. On 14 December 2000 a polar flare appeared on seven consecutive images. Comparison with previous images suggests that such transient bright features generally occur at the same location on the surface for similar CML, implying that they are mapping to approximately the same magnetic local time. The flares may be connected with bursty reconnection processes characterized by flux transfer events that were observed with the Pioneer and Voyager spacecraft at the dayside magnetopause.

[32] 7. It is shown that during this campaign, the probability for observing a bright auroral flare in the north (emitted power $\geq 1.5 \times 10^{11} \mathrm{~W}$ ) was less than $10 \%$, while there was a $30 \%$ chance to observe a moderate flare $(0.5-$ $\left.1.5 \times 10^{11} \mathrm{~W}\right)$.

[33] 8. Arc-like features have already been observed in past images and were referred to as the "equatorward surge." This arc is shown to move equatorward as the CML increases. It is suggested that the arc-like feature corresponds to the Dungey cycle magnetopause X-line.

[34] Acknowledgments. The authors would like to thank Margaret Kivelson for her useful comments on the dark polar region. The authors also thank two anonymous referees for their valuable comments and suggestions. This work is based on observations with the NASA/ESA Hubble Space Telescope, obtained at the Space Telescope Science Institute (STScI), which is operated by AURA, Inc. for NASA under contract NAS5-26555. The research was supported by grant GO-08657.01-A from the Space Telescope Science Institute. DG and JCG are supported by the Belgian Fund for Scientific Research (FNRS) and SWHC by PPARC Senior Fellowship PPA/N/S/2000/00197.

[35] Arthur Richmond thanks Tom Stallard and another reviewer for their assistance in evaluating this paper.

\section{References}

Ballester, G. E., et al., Time-resolved observations of Jupiter's far- ultraviolet aurora, Science, 274, 409-412, 1996.

Bunce, E. J., and S. W. H. Cowley, Divergence of the equatorial current in the dawn sector of Jupiter's magnetosphere, Planet. Space Sci., 49, $1089-1113,2001$

Clarke, J. T., et al., Hubble Space Telescope imaging of Jupiter's UV aurora during the Galileo orbiter mission, J. Geophys. Res., 103, 20,21720,236, 1998 .

Clarke, J. T., et al., Ultraviolet auroral emissions from the magnetic footprints of Io, Ganymede, and Europa on Jupiter, Nature, 415, 997-1000, 2002 .

Connerney, J. E .P., M. H. Acuña, N. F. Ness, and T. Satoh, New models of Jupiter's magnetic field constrained by the Io flux tube footprint, J. Geophys. Res., 103, 11,929-11,939, 1998.

Cowley, S. W. H., E. J. Bunce, T. S. Stallard, and S. Miller, Jupiter's polar ionospheric flows: Theoretical interpretation, Geophys. Res. Lett., 30(5), 1220, doi:10.1029/2002GL016030, 2003.

Dungey, J. W., Interplanetary field and the auroral zones, Phys. Rev. Lett., 6, 47-49, 1961

Gérard, J.-C., J. Gustin, D. Grodent, J. T. Clarke, and A. Grard, Spectral observations of transient features in the FUV Jovian polar aurora, J. Geophys. Res., 108(A8), 1319, doi:10.1029/2003JA009901, 2003.

Grodent, D., G. R. Gladstone, J.-C. Gérard, V. Dols, and J. H. Waite, Simulation of the morphology of the Jovian UV North Aurora observed with the Hubble Space Telescope, Icarus, 128, 306-321, 1997.

Grodent, D., J. H. Waite Jr., and J.-C. Gérard, A self-consistent model of the Jovian auroral thermal structure, J. Geophys. Res., 106, 12,933-12,952, 2001.

Grodent, D., J. T. Clarke, J. Kim, J. H. Waite Jr., and S. W. H. Cowley, Jupiter's main auroral oval observed with HST-STIS, J. Geophys. Res., 108, doi:10.1029/2003JA009921, in press, 2003

Hill, T. W., Inertial limit on corotation, J. Geophys. Res., 84, 6554-6558, 1979.

Hill, T. W., The Jovian auroral oval, J. Geophys. Res., 106, 8101-8107, 2001.

Huddleston, D. E., C. T. Russell, and G. Le, Magnetopause structure and the role of reconnection at the outer planets, J. Geophys. Res., 102, 24,289-24,302, 1997.

Knight, S., Parallel electric fields, Planet. Space Sci., 21, 741-750, 1973. Pallier, L., and R. Prangé, More about the structure of the high latitude Jovian aurorae, Planet. Space Sci., 49, 1159-1173, 2001.

Satoh, T., J. E. P. Connerney, and R. L. Baron, Emission source model of Jupiter's $\mathrm{H}_{3}^{+}$aurorae: A generalized inverse analysis of images, Icarus, $122,1-23,1996$

Southwood, D. J., and M. G. Kivelson, A new perspective concerning the influence of the solar wind on the Jovian magnetosphere, J. Geophys. Res. 106, 6123-6130, 2001

Stallard, T. S., S. Miller, G. Millward, and R. D. Joseph, On the dynamics of the jovian ionosphere and thermosphere 1 , The measurement of ion winds, Icarus, 154, 475-491, 2001.

Stallard, T. S., S. Miller, S. W. H. Cowley, and E. J. Bunce, Jupiter's polar ionospheric flows: Measured intensity and velocity variations poleward of the main auroral oval, Geophys. Res. Lett., 30, 1221, doi:10.1029/ 2002GL016031, 2003.

Vasyliunas, V. M., Plasma distribution and flow, in Physics of the Jovian Magnetosphere, edited by A. J. Dessler, p. 395, Cambridge Univ. Press, New York, 1983.

Waite, J. H., Jr., et al., An auroral flare at Jupiter, Nature, 410, 787-789, 2001.

Walker, J. H., Jr., and C. T. Russell, Flux transfer events at the Jovian magnetopause, J. Geophys. Res., 90, 7397-7405, 1985.

J. T. Clarke and J. Kim, CSP, Boston University, 725 Commonwealth Avenue, Boston, MA 02215, USA. (jclarke@bu.edu; juwhan@bu.edu)

S. W. H. Cowley, Department of Physics and Astronomy, Leicester University, Leicester, LE1 7RH, UK. (swhcl@ion.le.ac.uk)

J.-C. Gérard and D. Grodent, Institut d'Astrophysique et de Géophysique (B5c), Université de Liège, Allée du 6 août, 17, 4000 LIEGE, Belgium. (jc.gerard@ulg.ac.be; d.grodent@ulg.ac.be)

J. H. Waite Jr., Space Physics Research Laboratory, University of Michigan, 2436B SRB, Ann Arbor, MI 48109-2143, USA. (hunterw@) umich.edu) 